\title{
Spotlight on ixazomib: potential in the treatment of multiple myeloma
}

\author{
This article was published in the following Dove Press journal: \\ Drug Design, Development and Therapy \\ II January 2016 \\ Number of times this article has been viewed
}

\author{
Barbara Muz' \\ Rachel Nicole Ghazarian ${ }^{1,2}$ \\ Monica Ou ${ }^{1,3}$ \\ Micah John Luderer' \\ Hubert Daniel Kusdono ${ }^{1,2}$ \\ Abdel Kareem Azab' \\ 'Department of Radiation \\ Oncology, Cancer Biology Division, \\ Washington University in St Louis \\ School of Medicine, ${ }^{2}$ Department of \\ Pharmaceutical and Administrative \\ Sciences, St Louis College of \\ Pharmacy, ${ }^{3}$ Department of Biology, \\ St Louis University, St Louis, MO, USA
}

Correspondence: Abdel Kareem Azab Department of Radiation Oncology,

Cancer Biology Division, Washington University in St Louis School of Medicine, 45II Forest Park Avenue, Room 3103,

St Louis, MO 63108, USA

$\mathrm{Tel}+\mathrm{I} 3143629254$

Fax + I 3 | 43629790

Email aazab@radonc.wustl.edu

\begin{abstract}
Despite the significant therapeutic advances achieved with proteasome inhibitors (PIs) such as bortezomib and carfilzomib in prolonging the survival of patients with multiple myeloma, the development of drug resistance, peripheral neuropathy, and pharmacokinetic limitations continue to pose major challenges when using these compounds. Ixazomib is a second-generation PI with improved activity over other PIs. Unlike bortezomib and carfilzomib, which are administered by injection, ixazomib is the first oral PI approved by US Food and Drug Administration. This review discusses the biochemical properties, mechanisms of action, preclinical efficacy, and clinical trial results leading to the US Food and Drug Administration approval of ixazomib.
\end{abstract}

Keywords: proteasome inhibitor, oral administration, biological mechanism, clinical trials

\section{Introduction}

Multiple myeloma (MM) is a disorder of clonal cells that aggregate in the bone marrow (BM) stromal compartment. The MM cells are characterized by immune dysfunction, deregulated cell signaling, and high secretion of monoclonal protein detectable in the blood and/or urine. ${ }^{1,2} \mathrm{MM}$ primarily affects older individuals with a mean age of diagnosis between 63 years and 70 years of age. ${ }^{3}$ The disease constitutes $1.5 \%$ of all cancers and almost $15 \%$ of all hematological malignancies. There are $\sim 86,000$ new cases of MM occurring worldwide each year, with MM accounting for an estimated 11,090 deaths in the US in 2014 and an estimated 10,390 deaths in Europe in 2012. ${ }^{4}$ The introduction of the novel agents, including proteasome inhibitors (PIs) (bortezomib - Velcade and carfilzomib - Kyprolis) and immunomodulatory drugs (thalidomide - Thalomid, lenalidomide - Revlimid, and pomalidomide - Pomalyst), was an important advance in the therapy of MM., ${ }^{5,6}$ The combination regimens of these drugs and autologous stem cell transplantation substantially extended the average MM patient survival. ${ }^{3}$ Bortezomib was the first PI approved by the US Food and Drug Administration (FDA) in 2003 for relapsed/refractory MM patients, and also a few years later for treating newly diagnosed MM patients. ${ }^{7}$

Despite revolutionizing the treatment of MM, there are several limitations for the bortezomib treatment, including 1) not every MM patient will respond to bortezomib as a first-line therapy; 2) the majority of patients who initially respond favorably to bortezomib may later develop drug resistance and relapse; 3) peripheral neuropathy $(\mathrm{PN})$ is a dose-limiting toxicity of bortezomib, which can potentially result in permanent nerve damage to the extremities, ${ }^{8}$ and 4 ) other common side effects of bortezomib include fatigue, gastrointestinal effects, and modest cytopenias. ${ }^{8}$ Bortezomib is administered intravenously (iv) and recently subcutaneously (sc), which showed similar bioavailability with noninferior efficacy and a significantly lower incidence of 
PN compared to iv administration. ${ }^{9-12}$ Moreover, the safety profile of bortezomib was further improved by reducing dosing frequency from twice weekly to once weekly. ${ }^{13} \mathrm{~A}$ second-generation PI, carfilzomib, was approved by the US FDA in 2012 for the treatment of MM patients. Carfilzomib offered a therapeutic benefit to a fraction of patients who relapsed from bortezomib treatment while showing decreased PN. ${ }^{14}$ Nonetheless, drug resistance was also observed following carfilzomib treatment. ${ }^{15}$ One likely contributor to this resistance is the drug efflux pump P-glycoprotein (P-gp). Carfilzomib is a known P-gp substrate, and a few studies have demonstrated that carfilzomib-resistant cells exhibited increased P-gp expression. ${ }^{16-18}$ A significant limitation of the treatment with bortezomib and carfilzomib is that both these drugs are administered iv, which implies the need for health care professionals to administer the dose in a clinic or a hospital, which inevitably reduces the patient's quality of life and increases the treatment costs. ${ }^{19}$ Ixazomib (Ninlaro) is a small molecule PI that overcomes the aforementioned limitations of bortezomib and carfilzomib. In November 2015, ixazomib became the first orally administered PI to be approved by the US FDA. In this review, we highlight the chemical properties, biological activity, and mechanism of action of ixazomib, as well as summarize the clinical trials to test its efficacy in patients with MM.

\section{Chemical structure and mechanism of action of ixazomib}

Ixazomib (formerly known as MLN9708) was developed through a large-scale screening of boron-containing PIs with physiochemical properties distinct from bortezomib, in pursuit of efficacious PIs with increased efficacy and reduced side effects. ${ }^{20-22}$

Bortezomib structurally resembles dipeptide phenylalanine-leucine in which the terminal carboxylic acid is replaced by boric acid (Table 1 ). ${ }^{23}$ Bortezomib is a slowly reversible inhibitor of the $\beta 1$ caspase-like subunit and $\beta 2$ trypsin-like subunit, with preference to the $\beta 5$ chymotrypsinlike subunit of $20 \mathrm{~S}$ proteolytic site of proteasome..$^{21,24}$ Inhibition of the catalytic $\beta 5$ site occurs when the boric acid group forms covalent bound with the hydroxyl group of the $N$-terminal threonine residue. ${ }^{22,25}$ On the other hand, carfilzomib has a tripeptide backbone containing phenylalanine, leucine, and homophenylalanine with terminal epoxyketone group (Table 1). ${ }^{23,26}$ Carfilzomib is an irreversible inhibitor with high specificity for the $\beta 5$ chymotrypsin-like subunit of the proteasome. ${ }^{27}$ Ixazomib belongs to the same structural class and acts through the same mechanism as bortezomib.
Ixazomib consists of an alanine-leucine dipeptide core with a citrate-protected boric acid and preferentially inhibits the $\beta 5$ chymotrypsin-like subunit of proteasome, which cleaves proteins after hydrophobic residues. Furthermore, at high concentrations, ixazomib is capable of inhibiting other proteolytic sites of $20 \mathrm{~S}$ proteasome, including the $\beta 1$ caspase-like subunit, which cleaves proteins after acidic residues, and $\beta 2$ trypsin-like subunit, which cleaves after basic residues (Table 1 and Figure 1). ${ }^{22}$

Ixazomib is a citrate ester of boronic acid, which when exposed to aqueous solutions (eg, gastrointestinal tract and plasma) hydrolyzes to free boric acid metabolite MLN2238 that carries the biological effect (Figure 1). ${ }^{21}$ Xenograft mouse models demonstrated that ixazomib has significantly higher antitumor activity, which is attributed to distribution of larger blood volume compared to bortezomib, conferring greater pharmacodynamics. ${ }^{21,22}$ The clinical trials of ixazomib showed low incidence of PN compared to bortezomib, which may be ascribed to the high specificity of ixazomib in inhibiting the chymotrypsin-like site of the 20 S proteasome. ${ }^{21,28}$ Moreover, ixazomib elicits therapeutic response in patients who were bortezomib resistant. ${ }^{14,20}$

\section{Biological mechanisms of ixazomib Cell signaling pathways}

In vitro studies performed with different human MM cell lines treated with various concentrations of ixazomib showed a concentration-dependent decrease in the viability of all treated cell lines. Furthermore, it was shown that ixazomib induced apoptosis of MM cell lines resistant to conventional therapies and in primary MM cells obtained from bortezomiband lenalidomide-resistant patients. ${ }^{20}$ The apoptotic activity of ixazomib was mediated by the activation of caspase-8, caspase-9, and caspase-3 and other apoptotic pathways such as p53-p21, p53-NOXA-PUMA, and Rb-E2F. Ixazomib also induced the expression of binding immunoglobulin protein and CCAAT-enhancer-binding protein homologous protein transcription factor - both connected to endoplasmic reticulum stress response. ${ }^{20}$

MicroRNA profiling studies with MM cells showed that ixazomib induced upregulation of $m i R 33 b$, a tumor suppressor gene that is constitutively expressed at low levels in MM cells. Increased miR33b expression leads to cell death and apoptosis by blocking proto-oncogene (PIM-1) via inhibiting serine/threonine kinase activity. Furthermore, this increased expression is associated with reduced migration and viability of MM cells, as well as increased sensitivity to ixazomib. ${ }^{29}$ 


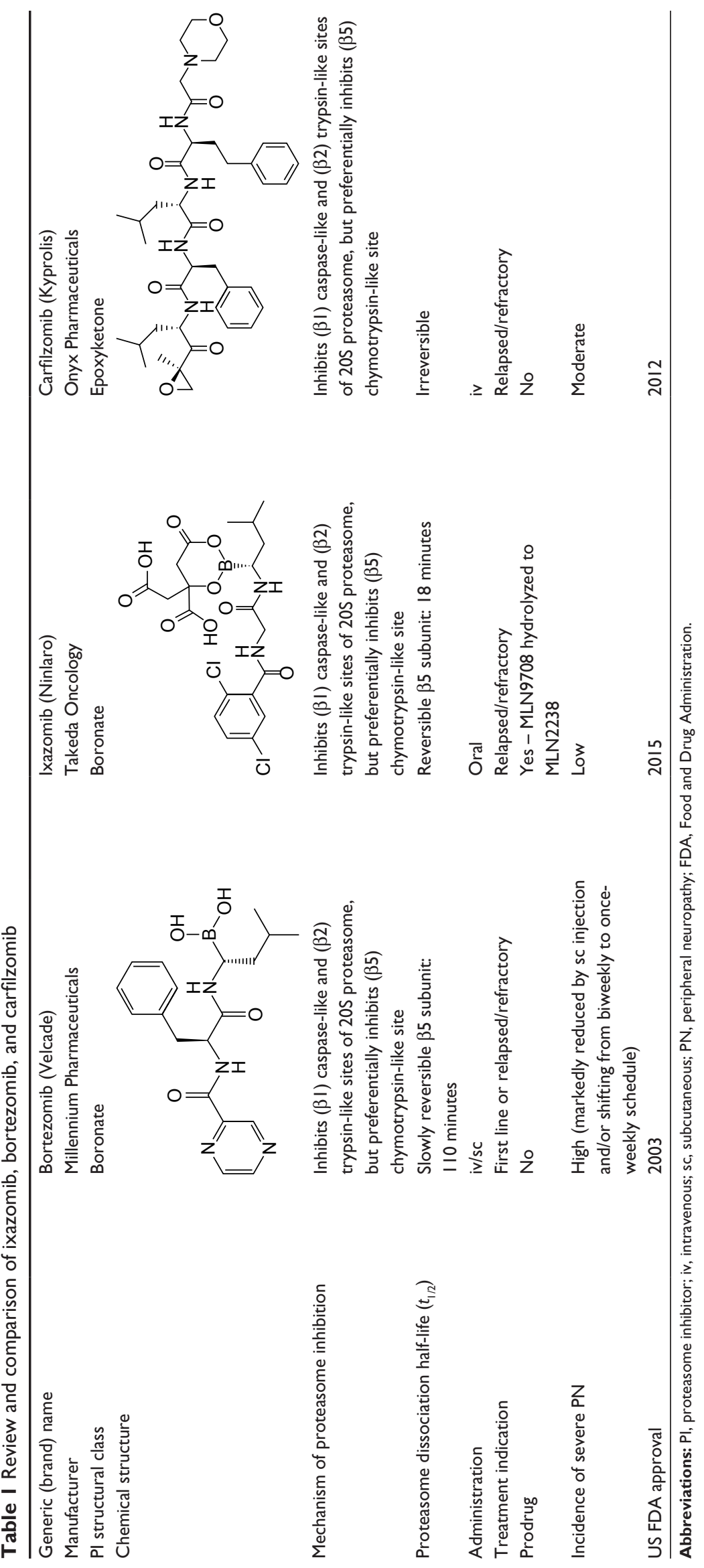




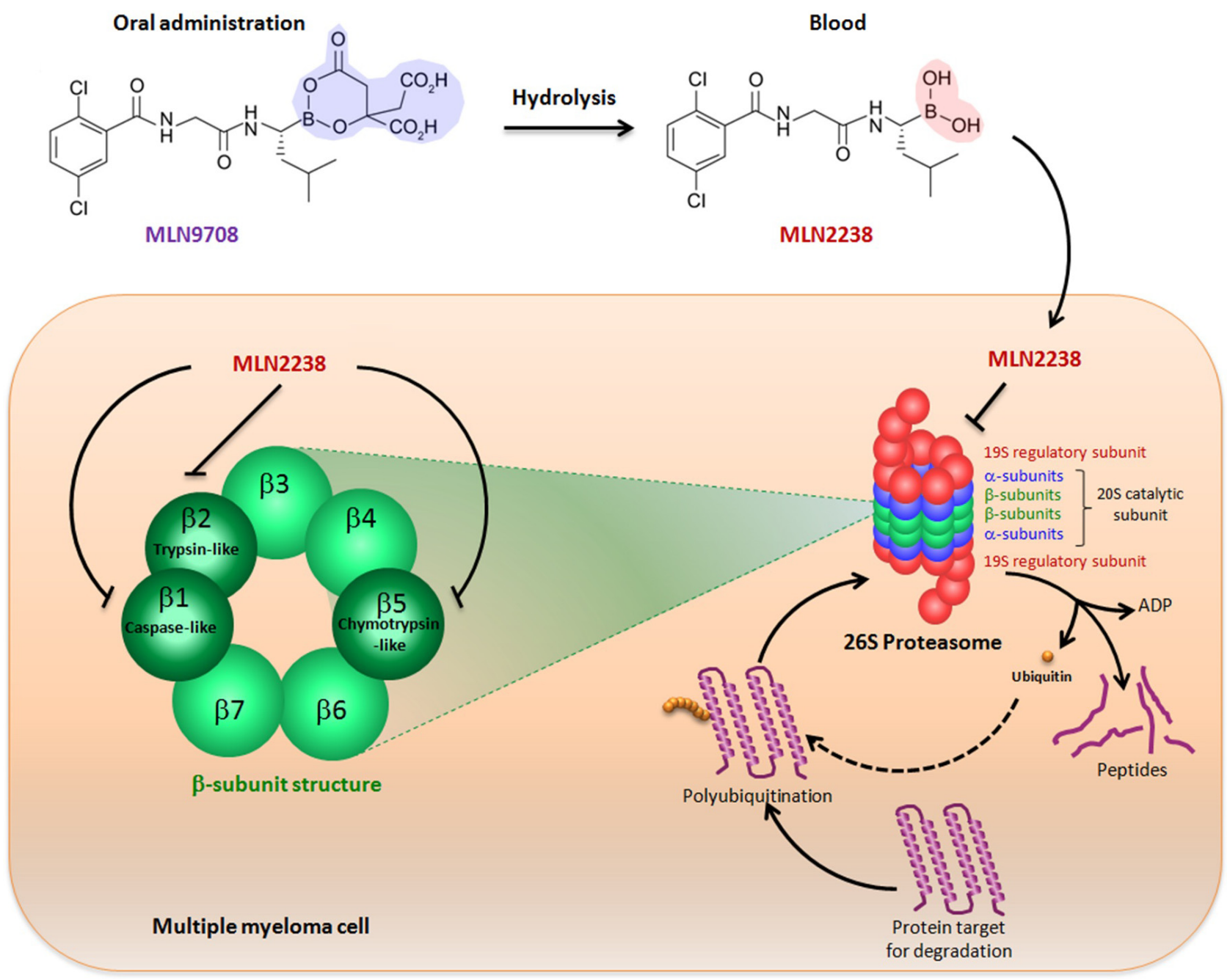

Figure I Mechanism of action of ixazomib.

Notes: Ixazomib (MLN9708) administered orally as a capsule is rapidly absorbed and hydrolyzed to the biologically active form (MLN2238) when it comes in contact with aqueous plasma. Ixazomib blocks protein degradation by inhibiting the 20S catalytic subunit of the 26S proteasome. More specifically, at lower concentrations, MLN2238 inhibits the $\beta 5$ chymotrypsin-like subunit, which cleaves proteins after hydrophobic residues. At high concentrations, MLN2238 inhibits the $\beta$ I caspase-like subunit and $\beta 2$ trypsin-like subunit, which cleave proteins after acidic and basic residues, respectively.

\section{Antitumor activity of ixazomib in vivo}

Treatment of MM-bearing mice with ixazomib induced a significant inhibition of MM tumor growth and a significant increase in survival compared to vehicle- and bortezomibtreated mice. In accordance with the in vitro data, when compared to vehicle-treated mice, ixazomib-treated mice showed an increase in the number of cleaved caspase-3-positive cells, increase in the number of TUNEL-positive cells, and decrease in the proliferation marker Ki-67. Immunostaining of harvested mouse tumors revealed that ixazomib inhibited the angiogenic activity of tumors and reduced the expression of angiogenesis markers such as vascular endothelial growth factor receptor 2 and platelet endothelial cell adhesion molecule, while displaying normal levels of creatinine, hemoglobin, and bilirubin. ${ }^{20}$

\section{Anti-BM microenvironment activity of ixazomib}

Acellular components include cytokines and growth factors, which facilitate cell proliferation, extracellular matrix, a scaffold promoting cell-cell interactions, and hypoxia niche, which causes limited oxygen diffusion as well as alters gene expression promoting drug resistance. ${ }^{30,31}$ Cellular components include stromal cells, which facilitate adhesion and proliferation, ${ }^{32-35}$ endothelial cells, which create blood vessels thus contribute to metastasis, ${ }^{36}$ and osteoblasts/ osteoclasts, which contribute to bone lytic lesions. ${ }^{37,38}$ In vitro, ixazomib inhibited the NF- $\kappa$ B pathway in MM stromal cells, reducing the release of cytokines that are vital for growth and survival of MM cells. Thus, treatment with ixazomib disrupts the cytoprotective effects of the BM 
microenvironment on MM cells and inhibits proliferation of MM cells. ${ }^{20}$

Osteolytic lesions are the most common complication of MM. ${ }^{39}$ It was demonstrated that ixazomib has a positive effect against MM-induced bone lytic lesions, since it inhibited osteoclast resorption with efficiency similar to bortezomib. It was demonstrated that early osteoclast differentiation was mediated by multiple signaling pathways that involve $\mathrm{NF}-\kappa \mathrm{B}$; ixazomib decreased NF- $\mathrm{KB}$ signaling in preosteoclasts by impairing the degradation of the cellular NF- $\kappa \mathrm{B}$ inhibitor, I- $\kappa \mathrm{B}$, by inhibiting the proteasome, which consequently reduced osteoclastogenesis. ${ }^{39}$ Moreover, in terms of osteoblast activity, ixazomib enhanced differentiation of osteoblast from primary mesenchymal stem cells isolated from myeloma and enhanced osteoblast functions. ${ }^{39}$

\section{Pharmacokinetic and pharmacodynamic parameters in animal models}

Biochemical analysis showed that the potency and selectivity of ixazomib and bortezomib to $\beta 1, \beta 2$, and $\beta 5$ subunits of proteasome are of the same magnitude, with preferential inhibitory activity towards $\beta 5$ subunit with the half maximal inhibitory concentration $\left(\mathrm{IC}_{50}\right)$ for ixazomib $3.4 \mathrm{nmol} / \mathrm{L}$ and for bortezomib $2.4 \mathrm{nmol} / \mathrm{L}$. The half-life $\left(t_{1 / 2}\right)$ of dissociation of ixazomib from the proteasome was found to be approximately six times shorter than that of bortezomib (18 minutes versus 110 minutes), which was consistent with the recovery of proteasome activity with bortezomib-treated cells recovering slower than ixazomib-treated cells. ${ }^{22}$

However, when administered iv, ixazomib was shown to have superior pharmacokinetic parameters compared with bortezomib; the maximal plasma concentration $\left(C_{\max }\right)$ of ixazomib was $17,000 \mathrm{ng} / \mathrm{mL}$ compared to $321 \mathrm{ng} / \mathrm{mL}$ for bortezomib. In addition, ixazomib provided a greater plasma exposure (area under the curve $\left[\mathrm{AUC}_{0-24 \mathrm{~h}}\right]=8,090 \mathrm{~h} \cdot \mathrm{ng} / \mathrm{mL}$ ) compared with bortezomib $\left(\mathrm{AUC}_{0-24 \mathrm{~h}}=485 \mathrm{~h} \cdot \mathrm{ng} / \mathrm{mL}\right)$, when both PIs were injected iv using their maximum tolerated doses. Moreover, ixazomib demonstrated five times higher drug distribution from blood into tissues supported by blood volume distribution, $V_{\mathrm{d}}$, of $20.2 \mathrm{~L} / \mathrm{kg}$ compared to $V_{\mathrm{d}}=4.3 \mathrm{~L} / \mathrm{kg}$ for bortezomib.

\section{Ixazomib in clinical trials \\ Phase I clinical trial}

\section{Study design}

Being the first oral PI, the clinical trials of ixazomib in patients with relapsed and/or refractory MM began with openlabel, Phase I dose-escalation studies and expansion cohort studies. ${ }^{19}$ In these studies, ixazomib was given twice weekly
(0.24-2.23 mg/m $\mathrm{m}^{2}$ on days $1,4,8$, and 11 of a 21 -day cycle) to 60 patients who met the following criteria: $>18$ years old with a measurable disease, an absolute neutrophil count $\geq 1,000$ cells $/ \mathrm{mm}^{3}$, platelet count $\geq 75,000$ cells $/ \mathrm{mm}^{3}$, a total bilirubin $\leq 1.5 \times$ the upper limit of normal, aspartate aminotransferase and alkaline aminotransferase $\leq 2.5 \times$ upper limit of normal, and creatinine clearance $\geq 20 \mathrm{~mL} / \mathrm{min}$ within 3 days of receiving the first dose. The exclusion criteria included uncontrolled preexisting comorbidities that may interfere with the study, as well as the previous treatment with a PI. Dose escalation of ixazomib was done in a standard $3+3$ scheme with the modified Fibonacci dose sequence. Investigators analyzed the dose-limiting toxicities that occurred in patients during cycle 1 in order to determine the maximum tolerated dose. ${ }^{19}$

\section{Toxicity and adverse effects}

Of the patients who remained on the maximum tolerated dose of $2.0 \mathrm{mg} / \mathrm{m}^{2}$, or an equivalent fixed dose of $3.7 \mathrm{mg}, \sim 62 \%$ experienced drug-related grade $\geq 3$ adverse events (AEs), $27 \%$ of whom were grade 4 . The most common drug-related AEs included nausea (42\%), thrombocytopenia (42\%), fatigue (40\%), skin and subcutaneous tissue disorders (40\%), vomiting (25\%), diarrhea (23\%), pyrexia (20\%), neutropenia (18\%), PN (12\%), and chills (10\%). Of the seven patients with drug-related $\mathrm{PN}$, three patients experienced grade $1 \mathrm{PN}$ at baseline, while others experienced a worsening of PN to grade 2 in cycle 2 at $0.8 \mathrm{mg} / \mathrm{m}^{2}$ in the cohort study, worsening from baseline while remaining at grade 1 in cycle 2 at $1.68 \mathrm{mg} / \mathrm{m}^{2}$ in the cohort study, or worsening grade 2 during cycle 3 of the relapsed and refractory cohort.

In the dose-escalation cohort with $2.0 \mathrm{mg} / \mathrm{m}^{2}$ ixazomib given twice weekly, a patient experienced spinal cord compression as a result of disease progression during cycle 1 . In the relapsed and refractory cohort studies, five people discontinued treatment due to drug-related thrombocytopenia during cycle 1, drug-related nausea and fatigue with PN during cycle 5, and pneumonia in cycle 2 as well as cycle 5 . Two dose-limiting toxicities of grade 3 rash and grade 4 thrombocytopenia occurred with $2.23 \mathrm{mg} / \mathrm{m}^{2}$ of ixazomib. ${ }^{19}$

\section{Pharmacokinetics and pharmacodynamics in Phase I clinical trials}

Ixazomib displayed rapid absorption with a maximal concentration time $\left(T_{\max }\right)$ of 1 hour, $C_{\max }$ of $7.78 \mathrm{ng} / \mathrm{mL}$, and a terminal $t_{1 / 2}$ of 3.3-7.4 days following a dose of $0.48 \mathrm{mg} / \mathrm{m}^{2}$. Linear pharmacokinetics was observed with a direct proportionality between the plasma concentration $(7.78-75.9 \mathrm{ng} / \mathrm{mL})$ and the administered dose (range of $0.48-2.23 \mathrm{mg} / \mathrm{m}^{2}$ ). The Phase I study showed that $2.0 \mathrm{mg} / \mathrm{m}^{2}$ of ixazomib administered 
orally twice weekly was the optimal dose for efficacy and AEs. This dose elicited a positive response and was effective in controlling the disease, while the AEs were reversible, minor, and manageable. ${ }^{19}$

\section{Phase II clinical trials Study design}

Open-label Phase II trials were initiated to test the safety, tolerability, and efficacy of ixazomib. ${ }^{7}$ Phase II trials also investigated the efficacy of the addition of dexamethasone to the ixazomib therapy. Patients were required to be at least 18 years, have relapsed MM after one or more attempted therapies, have a measurable disease (serum M-protein $\geq 1 \mathrm{~g} / \mathrm{dL}$ or urine M-protein $\geq 200 \mathrm{mg} / 24$ hours or involved free light chain level $\geq 10 \mathrm{mg} / \mathrm{dL}$ ), have an Eastern Cooperative Oncology Group performance status of $0-2$, absolute neutrophil count $\geq 1,000$ cells $/ \mathrm{mm}^{3}$, platelets $\geq 75,000$ cells/ $\mathrm{mm}^{3}$, and could not be on a CYP1A2 or CYP3A4 inducer/ inhibitor within 14 days of study. The patients were also excluded from the study, if they experienced any of the following disease states: uncontrolled cardiovascular conditions (hypertension, cardiac arrhythmias, congestive heart failure, unstable angina, or myocardial infarction within the past 6 months), HIV, positive hepatitis B surface antigen, hepatitis $\mathrm{C}$ infection, or patient allergy to any of the medications, analogs of the medications, or excipients that may interfere with the bioavailability of the drug. In total, 33 patients with relapsed MM were enrolled in this trial, and on average, the patients had previously received two alternative therapies.

The treatment goal was to receive an overall response rate of the drug using only a single agent in relapsed MM with no prior use of a PI or less than six cycles of therapy with a previous PI. The secondary goal was to obtain an overall response rate of the drug with the addition of dexamethasone. Patients in the Phase II study received, on average, eight cycles of therapy: 19 patients received four cycles, 16 patients received eight cycles, and the remaining 12 patients received 12 cycles. The median dose that 27 patients received per cycle was $12 \mathrm{mg}$ of ixazomib, ranging from $5.5 \mathrm{mg}$ to $15.6 \mathrm{mg}$. Dexamethasone was administered at the following occasions: 1) at the end of cycle 2,2) when patients showed no response to ixazomib alone by the end of cycle 4, or 3 ) if the patient experienced disease progression at any point during the treatment. ${ }^{7}$

\section{Toxicity and adverse effects}

AEs experienced by patients in the Phase II trial were identical to those experienced in the Phase I studies - 40\% experienced grade 3 or grade 4 and 19\% experienced grade 4 .
Common AEs included thrombocytopenia, fatigue, nausea, and diarrhea. No patients died during the study period. Drug-induced grade 1 and grade 2 PN was observed in eight patients and five patients, respectively. No hematological toxicity was recorded; however, three patients discontinued drug therapy due to elevated stringent complete response (sCR) and thrombotic thrombocytopenic purpura. ${ }^{7}$

\section{Efficacy}

As a single agent, ixazomib is therapeutically effective in treating patients with relapsed and refractory MM, with minimal rates of PN. Overall, the Phase II studies showed that $34 \%$ of patients experienced partial response or better during the entire trial without the addition of dexamethasone. Two additional patients experienced a minor response, and one patient had both CR and sCR. In all, 67\% of the patients were initiated on dual dexamethasone treatment, out of which, 17 patients were placed on the secondary therapy for not achieving a response with ixazomib alone and five patients were placed on the secondary therapy for progression of the disease. Those treated with the secondary therapy achieved a response rate of 34\% after the dexamethasone addition. Of the patients in cycle 4 , five patients experienced a positive response to treatment, three patients experienced a partial response to treatment, one patient achieved a complete response to therapy, and one patient achieved an $\mathrm{SCR}$. $^{7}$

A number of Phase II clinical trials are evaluating a three-drug regimen (or more) including ixazomib, lenalidomide, and dexamethasone for four cycles, followed by a randomized maintenance therapy with either ixazomib or lenalidomide in order to prolong the disease-free period in patients after stem cell transplantation (NCT02253316). Other randomized Phase I/II trials are testing a combination of ixazomib with or without pomalidomide and dexamethasone given together in treating patients with refractory MM (NCT02004275). In addition, ixazomib is being evaluated in previously untreated symptomatic myeloma patients with high risk of progression, where patients are treated in combination with cyclophosphamide and dexamethasone (NCT01864018; Table 2).

\section{Phase III clinical trial}

There are a number of ongoing Phase III clinical trials examining the efficacy of ixazomib on progression-free survival, overall survival, and overall response rate at different stages of MM. These trials are randomized, double blinded, multicenter, and placebo controlled. No results have been 


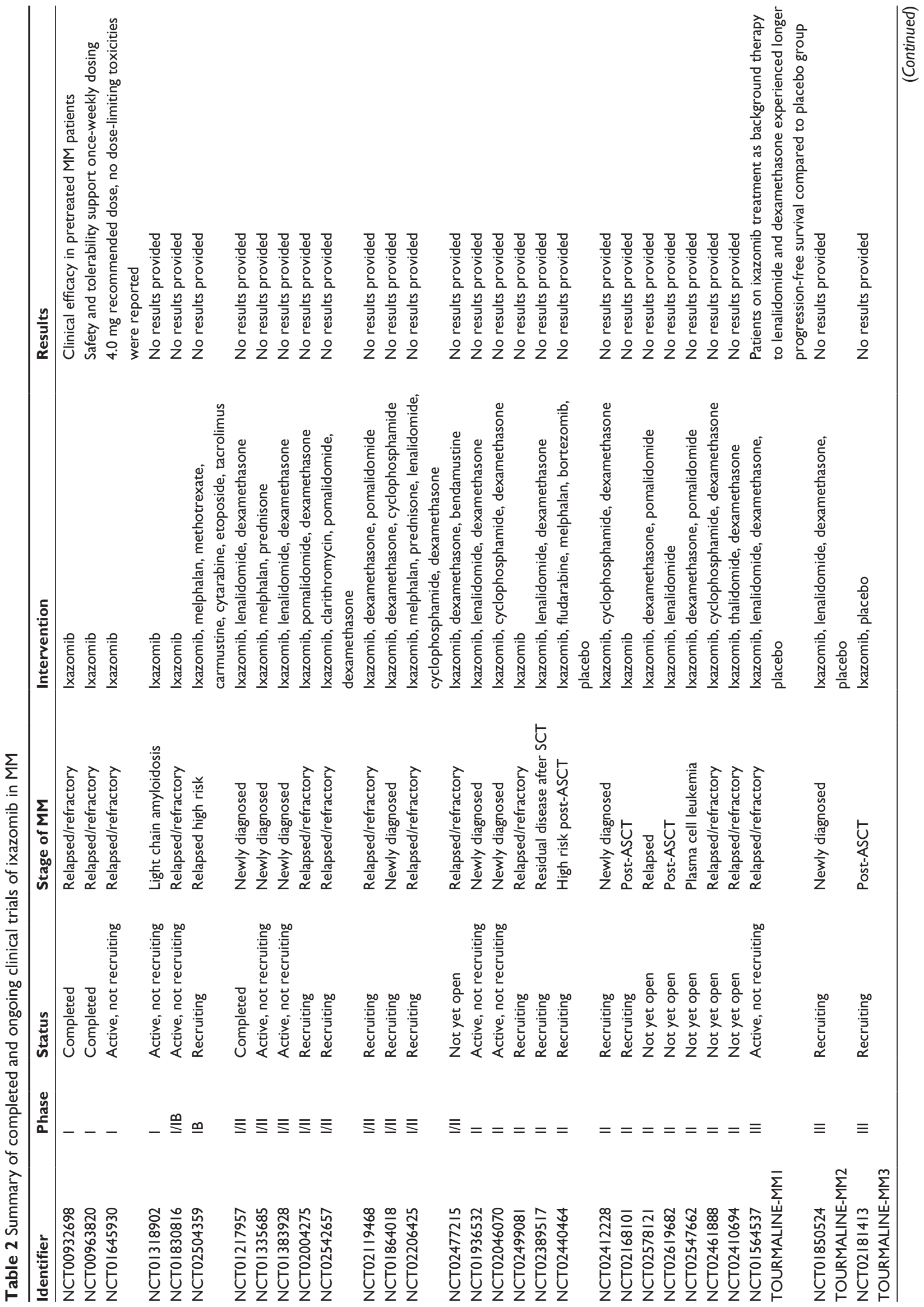


published yet. TOURMALINE-MM1 trial is being conducted in 837 relapsed/refractory MM patients (NCT01564537) and TOURMALINE-MM2 study in $>700$ newly diagnosed MM patients (NCT01850524). In these two studies, one patient group receives oral ixazomib $(4 \mathrm{mg})$ and the other group receives a placebo, while both groups receive lenalidomide and dexamethasone. Moreover, ixazomib ( $3 \mathrm{mg}$ ) as a single agent, compared to placebo, is being tested as a maintenance therapy in patients following autologous stem cell transplantation (TOURMALINE-MM3; NCT02181413) and in patients who have not received autologous stem cell transplantation (TOURMALINE-MM4; NCT02312258). A list of completed and ongoing clinical trials involving ixazomib in $\mathrm{MM}$ is given in Table 2.

\section{Conclusion}

Ixazomib is the first oral boronic acid-based PI approved by US FDA in combination with lenalidomide and dexamethasone for the treatment of MM patients who have received at least one prior therapy. Preclinical results demonstrated that ixazomib inhibits the chymotrypsin-like proteolytic ( $\beta 5)$ site of the 20S proteasome, reduces tumor progression by increasing $\mathrm{MM}$ cell apoptosis, and disrupts the interaction of MM cells with the BM microenvironment resulting in decreased angiogenesis and osteolytic lesions.

Currently, there are numerous clinical trials including five global Phase III trials on investigational oral ixazomib in $\mathrm{MM}$ and in other hematological malignancies such as lymphoma and amyloidosis, as well as in solid tumors. Based on study reports, ixazomib is therapeutically efficacious as a single agent but especially when used in combination with dexamethasone in the treatment of relapsed/refractory MM. Side effects are manageable and infrequent, including rash, lowered platelet and white blood cell counts, fatigue, diarrhea and nausea, and with a few instances of PN. By virtue of the convenience of oral administration, ixazomib is a promising option for MM treatment and is being continuously evaluated as a single agent or in combination with other therapies in Phase III trials for relapsed/refractory patients, newly diagnosed patients, and as a maintenance therapy.

\section{Acknowledgments}

This research was supported in part by the Washington University Institute of Clinical and Translational Sciences grant (UL1TR000448), the National Center for Advancing Translational Sciences (NCATS) of the National Institutes of Health (NIH), the National Cancer Institute of the NIH under Award Number U54CA199092, and the Multiple Myeloma 
Research Foundation. The content is solely the responsibility of the authors and does not necessarily represent the official view of the funding agencies.

\section{Disclosure}

Abdel Kareem Azab reports receiving commercial research grant from Selexys, Verastem, Karyopharm, and Cellworks and is the founder and owner of Targeted Therapeutics LLC and Cellatrix LLC. The other authors report no conflicts of interest in this work.

\section{References}

1. Cottini F, Anderson K. Novel therapeutic targets in multiple myeloma. Clin Adv Hematol Oncol. 2015;13(4):236-248.

2. Karp Leaf R, Cho HJ, Avigan D. Immunotherapy for multiple myeloma, past, present, and future: monoclonal antibodies, vaccines, and cellular therapies. Curr Hematol Malig Rep. Epub 2015 Sep 4.

3. Gentile M, Recchia AG, Mazzone C, et al. Emerging biological insights and novel treatment strategies in multiple myeloma. Expert Opin Emerg Drugs. 2012;17(3):407-438.

4. Moreau P, Touzeau C. Multiple myeloma: from front-line to relapsed therapies. Am Soc Clin Oncol Educ Book. 2015;35:e504-e511.

5. de la Puente P, Azab AK. Contemporary drug therapies for multiple myeloma. Drugs Today (Barc). 2013;49(9):563-573.

6. de la Puente P, Muz B, Azab F, et al. Molecularly targeted therapies in multiple myeloma. Leuk Res Treatment. 2014;2014:976567.

7. Kumar SK, LaPlant B, Roy V, et al. Phase 2 trial of ixazomib in patients with relapsed multiple myeloma not refractory to bortezomib. Blood Cancer J. 2015;4:e338.

8. Dou QP, Zonder JA. Overview of proteasome inhibitor-based anti-cancer therapies: perspective on bortezomib and second generation proteasome inhibitors versus future generation inhibitors of ubiquitin-proteasome system. Curr Cancer Drug Targets. 2014;14(6):517-536.

9. Moreau P, Pylypenko H, Grosicki S, et al. Subcutaneous versus intravenous administration of bortezomib in patients with relapsed multiple myeloma: a randomised, phase 3, non-inferiority study. Lancet Oncol. 2011;12(5):431-440.

10. Arnulf B, Pylypenko H, Grosicki S, et al. Updated survival analysis of a randomized phase III study of subcutaneous versus intravenous bortezomib in patients with relapsed multiple myeloma. Haematologica. 2012;97(12):1925-1928.

11. Lok A, Mocquard J, Bourcier J, et al. Subcutaneous bortezomib incorporated into the bortezomib-thalidomide-dexamethasone regimen as part of front-line therapy in the context of autologous stem cell transplantation for multiple myeloma. Haematologica. 2014;99(3): e33-e34.

12. Moreau P, Pylypenko H, Grosicki S, et al. Subcutaneous versus intravenous bortezomib in patients with relapsed multiple myeloma: subanalysis of patients with renal impairment in the phase III MMY3021 study. Haematologica. 2015;100(5):e207-e210.

13. Wang L, Wang KF, Chang BY, et al. Once-weekly subcutaneous administration of bortezomib in patients with multiple myeloma. Asian Pac J Cancer Prev. 2015;16(5):2093-2098.

14. Steele JM. Carfilzomib: a new proteasome inhibitor for relapsed or refractory multiple myeloma. J Oncol Pharm Pract. 2013;19(4):348-354.

15. Buac D, Shen M, Schmitt S, et al. From bortezomib to other inhibitors of the proteasome and beyond. Curr Pharm Des. 2013;19(22):4025-4038.

16. Ao L, Wu Y, Kim D, et al. Development of peptide-based reversing agents for p-glycoprotein-mediated resistance to carfilzomib. Mol Pharm. 2012;9(8):2197-2205.

17. Abraham J, Salama NN, Azab AK. The role of P-glycoprotein in drug resistance in multiple myeloma. Leuk Lymphoma. 2015;56(1):26-33.
18. Rumpold H, Salvador C, Wolf AM, et al. Knockdown of PgP resensitizes leukemic cells to proteasome inhibitors. Biochem Biophys Res Commun. 2007;361(2):549-554.

19. Richardson PG, Baz R, Wang M, et al. Phase 1 study of twice-weekly ixazomib, an oral proteasome inhibitor, in relapsed/refractory multiple myeloma patients. Blood. 2014;124(7):1038-1046.

20. Chauhan D, Tian Z, Zou B, et al. In vitro and in vivo selective antitumor activity of a novel orally bioavailable proteasome inhibitor MLN9708 against multiple myeloma cells. Clin Cancer Res. 2011;17(16): 5311-5321.

21. Offidani M, Corvatta L, Caraffa P, et al. An evidence-based review of ixazomib citrate and its potential in the treatment of newly diagnosed multiple myeloma. OncoTargets Ther. 2014;7:1793-1800.

22. Kupperman E, Lee EC, Cao Y, et al. Evaluation of the proteasome inhibitor MLN9708 in preclinical models of human cancer. Cancer Res. 2010;70(5):1970-1980.

23. Lednicer D. Antineoplastic Drugs: Organic Syntheses. Chichester: Wiley; 2015.

24. Adams J, Behnke M, Chen S, et al. Potent and selective inhibitors of the proteasome: dipeptidyl boronic acids. Bioorg Med Chem Lett. 1998; 8(4):333-338.

25. Touchet S, Carreaux F, Carboni B, et al. Aminoboronic acids and esters: from synthetic challenges to the discovery of unique classes of enzyme inhibitors. Chem Soc Rev. 2011;40(7):3895-3914.

26. Kim KB, Crews CM. From epoxomicin to carfilzomib: chemistry, biology, and medical outcomes. Nat Prod Rep. 2013;30(5):600-604.

27. Kuhn DJ, Chen Q, Voorhees PM, et al. Potent activity of carfilzomib, a novel, irreversible inhibitor of the ubiquitin-proteasome pathway, against preclinical models of multiple myeloma. Blood. 2007;110(9): 3281-3290.

28. Kumar SK, Berdeja JG, Niesvizky R, et al. Safety and tolerability of ixazomib, an oral proteasome inhibitor, in combination with lenalidomide and dexamethasone in patients with previously untreated multiple myeloma: an open-label phase 1/2 study. Lancet Oncol. 2014;15(13): $1503-1512$.

29. Tian Z, Zhao JJ, Tai YT, et al. Investigational agent MLN9708/2238 targets tumor-suppressor miR33b in MM cells. Blood. 2012;120(19): 3958-3967.

30. Azab AK, Hu J, Quang P, et al. Hypoxia promotes dissemination of multiple myeloma through acquisition of epithelial to mesenchymal transition-like features. Blood. 2012;119(24):5782-5794.

31. Kawano Y, Moschetta M, Manier S, et al. Targeting the bone marrow microenvironment in multiple myeloma. Immunol Rev. 2015;263(1): $160-172$.

32. Azab AK, Runnels JM, Pitsillides C, et al. CXCR4 inhibitor AMD3100 disrupts the interaction of multiple myeloma cells with the bone marrow microenvironment and enhances their sensitivity to therapy. Blood. 2009;113(18):4341-4351.

33. Azab AK, Sahin I, Moschetta M, et al. CXCR7-dependent angiogenic mononuclear cell trafficking regulates tumor progression in multiple myeloma. Blood. 2014;124(12):1905-1914.

34. Azab AK, Quang P, Azab F, et al. P-selectin glycoprotein ligand regulates the interaction of multiple myeloma cells with the bone marrow microenvironment. Blood. 2012;119(6):1468-1478.

35. Azab AK, Azab F, Blotta S, et al. RhoA and Rac1 GTPases play major and differential roles in stromal cell-derived factor-1-induced cell adhesion and chemotaxis in multiple myeloma. Blood. 2009;114(3):619-629.

36. Ghobrial IM. Myeloma as a model for the process of metastasis: implications for therapy. Blood. 2012;120(1):20-30.

37. Toscani D, Bolzoni M, Accardi F, et al. The osteoblastic niche in the context of multiple myeloma. Ann N Y Acad Sci. 2015;1335:45-62.

38. Roodman GD. Pathogenesis of myeloma bone disease. Leukemia. 2009; 23(3):435-441.

39. Garcia-Gomez A, Quwaider D, Canavese M, et al. Preclinical activity of the oral proteasome inhibitor MLN9708 in Myeloma bone disease. Clin Cancer Res. 2014;20(6):1542-1554. 


\section{Publish your work in this journal}

Drug Design, Development and Therapy is an international, peerreviewed open-access journal that spans the spectrum of drug design and development through to clinical applications. Clinical outcomes, patient safety, and programs for the development and effective, safe, and sustained use of medicines are a feature of the journal, which

has also been accepted for indexing on PubMed Central. The manuscript management system is completely online and includes a very quick and fair peer-review system, which is all easy to use. Visit http://www.dovepress.com/testimonials.php to read real quotes from published authors.

Submit your manuscript here: http://www.dovepress.com/drug-design-development-and-therapy-journal 